\title{
Strong-field photoemission from silicon field emitter arrays
}

\author{
Phillip D. Keathley ${ }^{1}$, Alexander Sell ${ }^{1,2}$, William P. Putnam, Stephen Guerrera ${ }^{3}$, Luis Velásquez-García ${ }^{3}$, \\ and Franz X. Kärtner ${ }^{1,2, *}$
}

Received 16 July 2012, revised 23 October 2012, accepted 24 October 2012

Published online 13 December 2012

Strong-field photoemission from silicon field emitter arrays is investigated experimentally and results are explained using a "simple-man" optical-field emission model. Spectra are collected throughout an in-situ laser annealing process, leading to a red-shift in emitted electron energy along with an increase in electron yield. After the annealing process, a high energy plateau is formed which is explained through optical-field emission along with electron re-scattering with the tip surface.

\section{Introduction}

Strong-field photoemission of electrons, also called optical-field emission, is typically associated with gasphase atoms, however the study of similar photoemission processes from solid nanostructures has recently gained interest. [1-7] A major advantage to using a solid material to study strong-field emission is that the work functions of most metals and semiconductors are a factor of 3-5 lower than the ionization potential of typical noble gases. In addition, field enhancement through sharp tips and plasma oscillations further reduce the necessary incident intensity levels needed for tunneling emission. Strong-field tunneling emission from such structures is interesting in that it enables the generation of electron wavepackets whose duration is just a fraction of the driving laser cycle. Potential applications for such short, structured electron bursts range from ultrafast electron diffraction and microscopy [8], to free electron laser sources. An example of the latter application is found in [9], where it is proposed that the transverse structure of the electron current from a nano-tip array can be used to produce a longitudinally modulated electron beam for the direct generation of x-rays. Thus, the experimental investigation presented here is a first step towards unraveling the electron emission properties of large scale silicon nano-tip arrays as potential candidates for photocathodes in advanced x-ray sources.

Most studies to date have concentrated on laser driven emission from single metallic nano-tips, such as tungsten or gold. $[1,10]$ Nano-tips are advantageous as the electric field is highly enhanced at their apex, especially for plasmonic materials. [11] This enables access to strong-field ionization regimes and high field-emission yield without the use of high power laser beams, mitigating unwanted thermal emission and material damage.

An alternative to the common metals used is heavily n-doped silicon which has practical advantages when fabricating large scale emitter structures. First, scalable processes for creating highly uniform field emitter arrays have already been studied for the case of DC emission in silicon. $[12,13]$ Furthermore, silicon does not require highly stringent vacuum conditions for use as a field emitter (see for example the degradation of tungsten tips over time during photoemission as in [1]).

The sections that follow describe an experimental study of photo-electron emission energy spectra from an array of sharp silicon nanotips. In Section 2 an overview of the experimental setup is given. In Section 3 the experimental results are provided with further analysis and theoretical modeling of the photoemission process. Finally, Section 4 highlights the key conclusions of the work.

\footnotetext{
* Corresponding author E-mail: kaertner@)mit.edu

1 Department of Electrical Engineering and Computer Science and Research Laboratory of Electronics, Massachusetts Institute of Technology, 77 Massachusetts Avenue, Cambridge, MA 02139, USA

2 Center for Free-Electron Laser Science, DESY and Dept. of Physics University of Hamburg, Notkestraße 85, D-22607 Hamburg, Germany

3 Microsystems Technology Laboratories, Massachusetts Institute of Technology
} 


\section{Experimental setup}

The tips used in this experiment were fabricated from highly doped single-crystal silicon similar to those demonstrated in [13]. The tips are placed on a $10 \mu \mathrm{m}$ square pitch, each having an end radius of curvature ranging from 5 to $10 \mathrm{~nm}$. It is important to note here that the thin native oxide of the tips was not removed before the experiment.

The tips were illuminated at a grazing incidence of roughly $84^{\circ}$ with an $800 \mathrm{~nm}, 35$ fs laser beam focused to a spot of radius $80 \mu \mathrm{m}$. The laser polarization was TM with respect to the sample. For all of the spectra reported here, laser intensities of $5 \times 10^{10}$ to $1.6 \times 10^{11} \mathrm{~W} / \mathrm{cm}^{2}$ in the free-space focus were used.

Electron energy spectra were collected using a time of flight (TOF) electron spectrometer. This consists of a field-free $\mu$-metal shielded drift region of $\approx 35 \mathrm{~cm}$ in length followed by a multi-channel plate (MCP) detector. At high bias potentials the MCP can detect single electron events, with a rise time of $500 \mathrm{ps}$. The electron signal is collected and averaged using a serial data analyzer with a sample resolution of $50 \mathrm{ps}$, thus the fundamental time resolution is limited by the MCP, leading to an energy resolution of $0.05 \mathrm{eV}$ around a central energy of $10 \mathrm{eV}$. The time to energy conversion of the spectrometer is calibrated by comparing photo-electron energy spectra of high harmonic XUV radiation in Ar to the original high harmonic XUV photon spectrum. Since the ionization potential of Ar is well defined, and HHG XUV radiation forms sharp peaks spaced evenly by $2 \hbar \omega$, a photoelectron energy "ruler" is formed that is then used for calibration of the TOF spectrometer. A schematic of the experimental setup is shown in Figure 1.

For the data collection, spectra were taken at five different peak incident intensities, ranging from $5 \times$ $10^{10} \mathrm{~W} / \mathrm{cm}^{2}$ to $1.6 \times 10^{11} \mathrm{~W} / \mathrm{cm}^{2}$ in the free-space focus of the laser beam. While testing electron spectra from the tips at various spots along the sample, a distinct, repeatable annealing process was observed when applying laser intensities above a certain threshold. To study this laser induced annealing effect, after each round of taking spectra at low incident intensities, the pulse energy was increased above the observed annealing threshold. The location was then illuminated with this annealing energy for a fixed duration of time before once again taking spectra at the lower intensity values for comparison.

\section{Results and analysis}

Figure 2 shows the effects of the anealing process on the tips. Each spectrum is taken at a peak intensity of

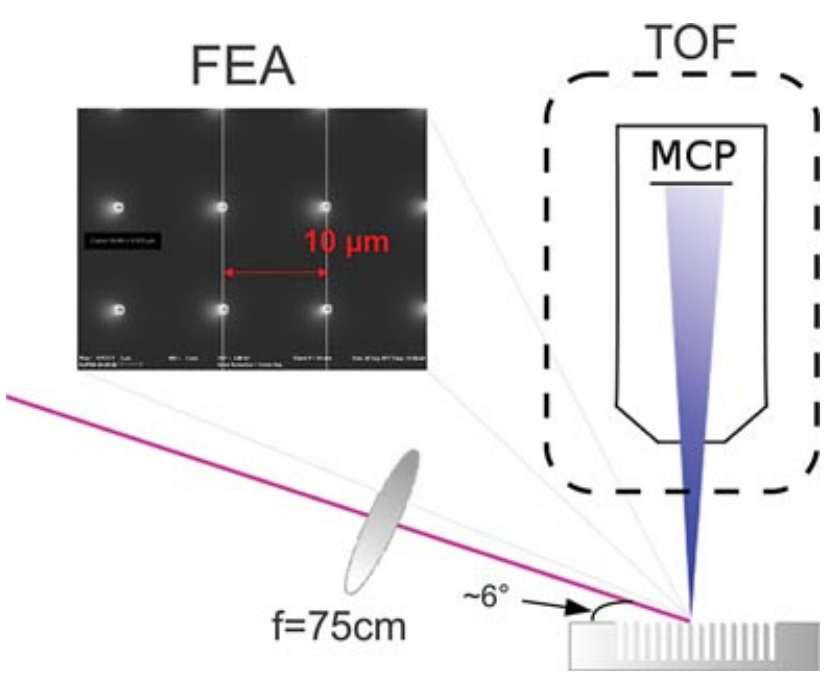

Figure 1 (online color at: www.ann-phys.org) Schematic of the experimental setup used. MCP: micro-channel plate detector. TOF: time of flight electron spectrometer. FEA: field emitter array. Input laser is a $35 \mathrm{fs}$ pulse centered around $800 \mathrm{~nm}$, having a focused spot size of $w_{0}=80 \mathrm{~nm}$ and a repetition rate of $1 \mathrm{kHz}$. Spectra were collected with unenhanced peak intensities ranging from $5 \times 10^{10} \mathrm{~W} / \mathrm{cm}^{2}$ to $1.6 \times 10^{11} \mathrm{~W} / \mathrm{cm}^{2}$, corresponding to unenhanced peak fields ranging from $0.6 \mathrm{~V} / \mathrm{nm}$ to $1.1 \mathrm{~V} / \mathrm{nm}$.

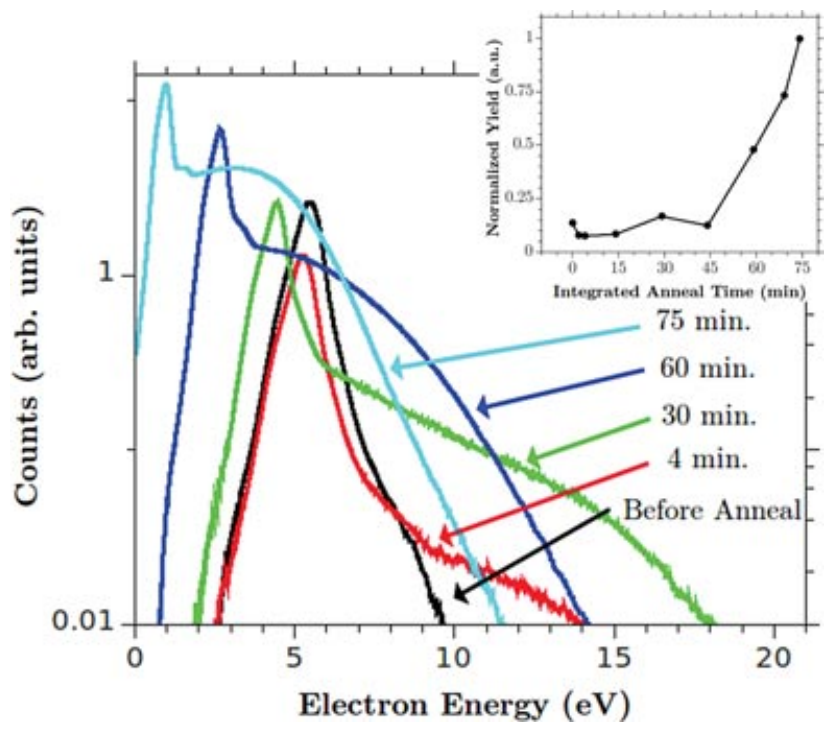

Figure 2 (online color at: www.ann-phys.org) Electron spectra taken as a function of increased anneal time at an incident pulse energy of $0.6 \mu \mathrm{J}$ incident energy. The times given are the integrated anneal times, starting with an oxidized position on the sample at time 0 . The annealing energies ranged from 1 to $2 \mu \mathrm{J}$ focused onto the same spot the spectra were taken from. Note the red shift of the main spectral peak. The inset shows integrated current yield as a function of anneal time. Initially there is a decrease in total electron yield before an eventual increase as anneal time is increased. 
$\underset{\substack{\text { anmalen } \\ \text { derp }}}{\text { physik }}$

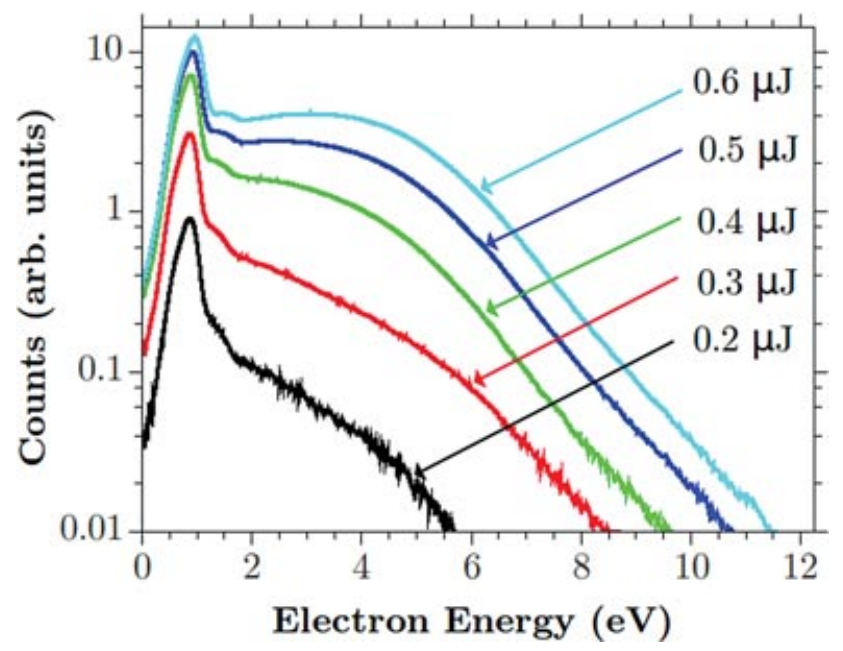

Figure 3 (online color at: www.ann-phys.org) Experimental results from the power scaling study of the same spot after the final integrated anneal step has been performed. The main spectral shows little change with increasing pulse energy. A significant plateau forms at high pulse energies, extending to higher energies with increased laser field strength.

$1.6 \times 10^{11} \mathrm{~W} / \mathrm{cm}^{2}$ at the given accumulated anneal time. Three main trends are clear. First, the formation of a higher energy plateau that is not prevalent before the anneal. Secondly, there is a net red-shift of the main peak in each spectrum, from an original value of $\approx 5 \mathrm{eV}$ before the anneal, to a value of $\approx 1 \mathrm{eV}$ after the last anneal step. Finally, the total electron yield varies over the anneal, first slightly decreasing before finally increasing.

Figure 3 shows a power scaling study after the total annealing process to demonstrate how the plateau scales with increased intensity, while the main spectral peak stays roughly fixed. This was true after all anneal times throughout the experiment. These features are both comparable to those already observed by others when performing energy-resolved photoemission experiments on metallic tips. $[1,5,14]$ The plateau is typically ascribed to re-scattering of the electron from the tip surface due to acceleration from the long-wavelength laser field driving the tunneling process.

A further justification that the strong field tunneling regime is achieved is found in the total current yield as a function of incident energy. A distinct slope change in yield versus incident energy is present after most of the anneal phases, such as the one plotted in Figure 4, which shows the integrated current vs. incident pulse energy after the final annealing step. A slope change occurs near an incident pulse energy of $0.4 \mu \mathrm{J}$. Others have found such a slope change should occur near a Keldysh param-
P. D. Keathley et al.: Strong-field photoemission from silicon field emitter arrays

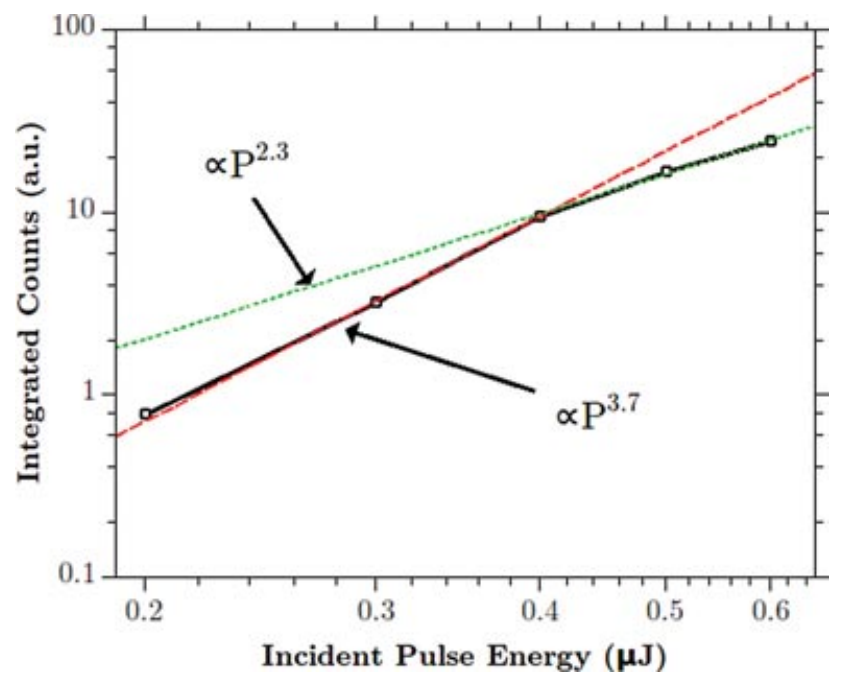

Figure 4 (online color at: www.ann-phys.org) Plot of total MCP current yield with increasing incident energy. The slope transition is typical of a transition from multiphoton emission to the strongfield regime, occurring near $\gamma \approx 2$. The black circles indicate the measured values. The red and green trend-lines show the power law relation before and after the slope change respectively, where $P$ is the pulse energy.

eter of $\gamma=\sqrt{\phi / 2 U_{p}} \approx 2$ [10], where $\phi$ is the work function of the tips, $U_{p}=q^{2} F_{\text {peak }}^{2} / 4 m \omega^{2}$ is the ponderamotive potential of the driving laser field, $q$ and $m$ the electron charge and mass, and $\omega$ and $F$ the laser frequency and field. For Keldysh parameters near and below this value, the emission approaches the quasi-static regime where it is dominated by electron tunneling, and the tunneling rate can be well approximated by substituting the laser's electric field directly into the tunneling rate equation for a static field (e.g. the Fowler-Nordheim tunneling equation). [15]

Considering the slope transition point observed along with the plateau feature extent, possible tip enhancement factors range from 10-14 giving a peak field strength range of 11-15.4 V/nm. This observation is reasonable when compared to electric field models of similar silicon structures at slightly lower wavelengths. [16] To compare our experimental results to theory, we turn to what is known as the "simple-man" model, commonly used with strong-field tunneling processes in gases [17], using a similar approach to [5].The basic overview of the model is described as follows.

1. An electron is emitted at time $t_{0}$ from the tunneling exit, with a probability determined by the tunneling rate $\Gamma$. 


\section{Vacuum}

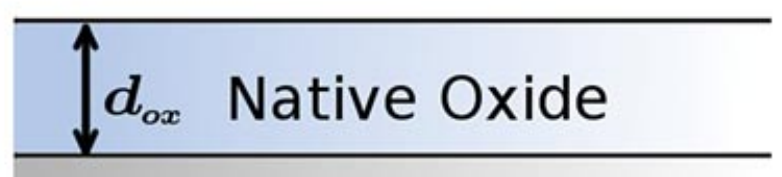

Silicon

(a)
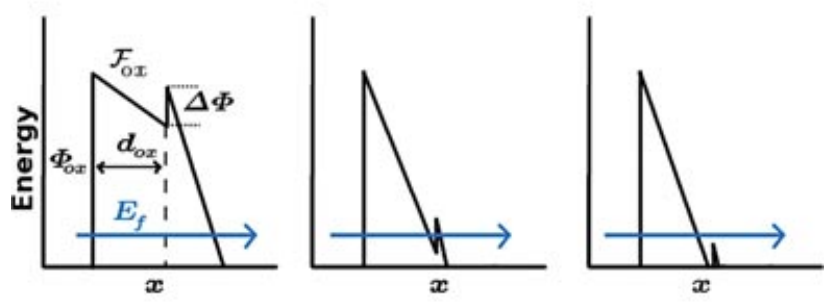

(b)

Figure 5 (online color at: www.ann-phys.org) In (a) the material stack at the surface of the tip is shown with native oxide present. In (b) the different tunneling scenarios are shown. As $F_{o x}$ is increased, the electron eventually escapes the first barrier and tunnels through the small barrier left from $\Delta \phi$. At even higher $F_{o x}$ the electron can tunnel directly through the oxide barrier into the continuum. These transitions are modeled with the Heaviside functions in equations (2) and (3)

2. The electron is then accelerated away from the tip by the electric field of the laser. In this part the potential of the tip is ignored, and the classical trajectories are solved.

3. If the electron returns to the tip, it is elastically scattered from the surface with some probability $R$.

For the tunneling rates, a modified Fowler-Nordheim (FN) rate equation is used, following the procedure found in [18] in order to account for the effects of any oxide layer. Rather than the situation where a single step barrier is present (no oxide), for the scenario of a thin oxide, a double step barrier is formed. The intermediate work function $\phi_{o x}$ is taken to be $3.5 \mathrm{eV}$ for the $\mathrm{Si}$ to $\mathrm{SiO}_{2}$ interface, while $\phi_{S i}=\phi_{o x}+\Delta \phi$ is taken to be $4.05 \mathrm{eV}$, the electron affinity of Si. The schematic of the barriers and different tunneling scenarios are shown in Figure 5. Ap- plying the WKB approximation to such a barrier leads to the following rate equation.

$$
\begin{aligned}
\Gamma \propto J= & \Theta(F(t)) \frac{q^{3}|F(t)|^{2}}{16 \pi^{2} \hbar \phi_{S i} B^{2}}\left(\frac{m^{*}}{m}\right) \\
& \times \exp \left(\frac{4 \sqrt{2 m}}{3 q \hbar|F(t)|} \phi_{S i}^{3 / 2} C\right)
\end{aligned}
$$

Here $B$ and $C$ are defined below.

$$
\begin{aligned}
B= & \epsilon_{o x} \sqrt{\frac{m_{o x}}{m}}\left[\left(\frac{\phi_{o x}}{\phi_{S i}}\right)^{1 / 2}\right. \\
& \left.-\Theta\left(\phi_{o x}-q\left|F_{o x}(t)\right| d_{o x}\right)\left(\frac{\phi_{o x}-q\left|F_{o x}(t)\right| d_{o x}}{\phi_{S i}}\right)^{1 / 2}\right] \\
& +\Theta\left(\phi_{S i}-q\left|F_{o x}(t)\right| d_{o x}\right)\left(1-\frac{q\left|F_{o x}(t)\right| d_{o x}}{\phi_{S i}}\right)^{1 / 2} \\
C= & \epsilon_{o x} \sqrt{\frac{m_{o x}}{m}}\left[\left(\frac{\phi_{o x}}{\phi_{S i}}\right)^{3 / 2}\right. \\
& \left.-\Theta\left(\phi_{o x}-q\left|F_{o x}(t)\right| d_{o x}\right)\left(\frac{\phi_{o x}-q\left|F_{o x}(t)\right| d_{o x}}{\phi_{S i}}\right)^{3 / 2}\right] \\
& +\Theta\left(\phi_{S i}-q\left|F_{o x}(t)\right| d_{o x}\right)\left(1-\frac{q\left|F_{o x}(t)\right| d_{o x}}{\phi_{S i}}\right)^{3 / 2}
\end{aligned}
$$

$\Theta(x)$ is the Heaviside function, $\epsilon_{o x}$ the dielectric constant of the oxide at $800 \mathrm{~nm}, d_{o x}$ the oxide thickness, $F(t)$ is the enhanced electric field of the laser in vacuum just above the $\mathrm{SiO}_{2}$ surface, and $F_{o x}(t)=F(t) / \epsilon_{o x}$ is the component of the electric field in the oxide. As in the experiment, the electric field is polarized along the direction of the tips. Here we also take $m^{*}$ as the effective electron mass in Si and $m_{o x}$ the effective mass in the oxide, $m$ to be the mass of an electron and $q$ the fundamental charge. Note that when $d_{o x}$ is set to zero, or the oxide properties are set to that of the vacuum, B and C both go to 1 and the rate equation reduces to that of traditional FN theory as expected.

The electric field was modeled with an enhancement factor having a dipolar decay away from the tip as in [5]. It is important here to simulate the spatial extent of the field enhancement as the tip radius of curvature, and thus the rate of field decay along axis, is on the order of the excursion length of the electron within the field during acceleration (several $\mathrm{nm}$ ). For the electron acceleration, a Runge-Kutta differential equation solver was used to determine the classical trajectories of the electron within the electric field after emission. Each trajectory is weighted by $\Gamma$ according to the electron birth time, and the electron is then modeled to have zero initial 
$\underset{\substack{\text { anmalen } \\ \text { derp }}}{\text { physik }}$

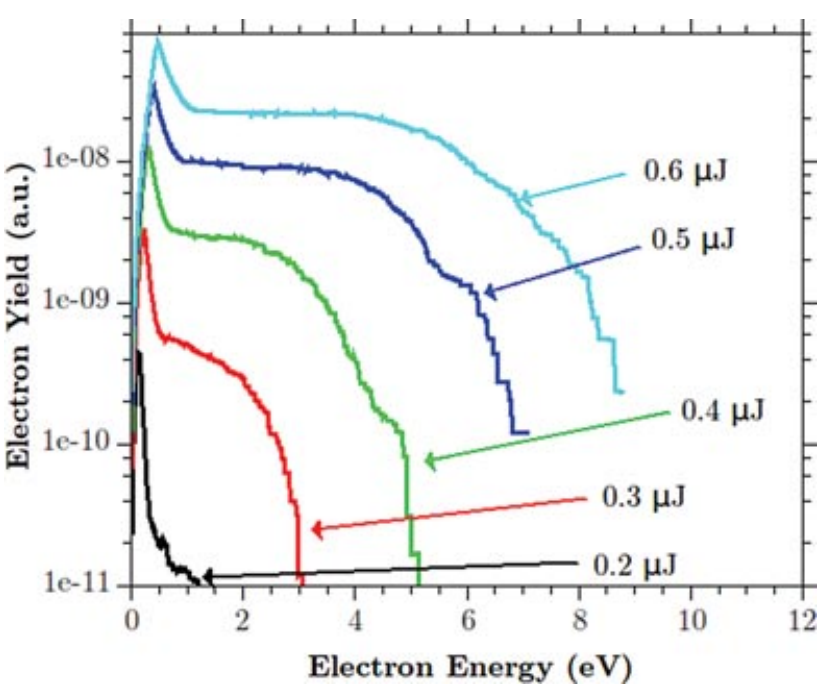

Figure 6 (online color at: www.ann-phys.org) Model results when simulating the experimental incident power study. The spectral features qualitatively match those of the experiment, each spectra having a sharp low energy peak with a broad plateau. The plateau can be turned on or off in the model through the electron rescattering probability, and scales with incident energy similar to the experimental data.

kinetic energy and an initial position at the tunnel exit. Scattered electrons are modeled as elastically scattering off of the silicon surface, traveling through the $\mathrm{SiO}_{2}$, since the scattering length of the electron within the oxide is on the order of $3 \mathrm{~nm}$ [19], which is greater than a typical native oxide.

When calculating the electron trajectories, spacecharge effects are deemed negligible. This is supported by the experimental results as the main spectral peak does not suffer energy broadening even with a significant increase in emitted current as one would expect when space charge effects are dominant. Furthermore, multiple re-scattering events are deemed negligible. The electric field used for calculating the trajectories in vacuum is represented by

$F(z, t)=F_{0}(t)\left[(\alpha-1)\left(\frac{\beta}{z+\beta}\right)^{3}+\exp \left\{\frac{-2 \log (2) z^{2}}{4 w_{0}^{2}}\right\}\right]$,

where $F(z, t)$ is the enhanced electric field, $z$ is the distance away from the top surface of the $\mathrm{SiO}_{2}, F_{0}(t)$ is the unenhanced on-axis field component, $\alpha$ is the field enhancement factor, $\beta$ is the tip radius of curvature and $w_{0}$ gives the unenhanced field waist.

Figure 6 gives the results of the theoretical model of the power scaling experiment. The spectra were averaged
P. D. Keathley et al.: Strong-field photoemission from silicon field emitter arrays

over different peak field values, the average value taken to be $12 \mathrm{~V} / \mathrm{nm}$. A native oxide thickness of $0.5 \mathrm{~nm}$ was used, along with a roughly fitted reflection probability of $R=0.3$. By turning off the reflectivity it can be shown that the sharp spectral peak is due purely to the electron trajectories that do not re-scatter, and the plateau feature is due to those that do. While a free electron with no scattering can only pick up a maximum of $2 U_{p} \approx 2.8 \mathrm{eV}$ from the driving laser field in our case at an incident of $0.6 \mu \mathrm{J}$, a re-scattered electron's energy can exceed $10 U_{p}[20]$, explaining the large energy extension observed.

While many qualitative effects are explained through the model, the annealing process is still not fully understood. Even after the final anneal step, the energy of the main spectral peak still differs from simulations by around $0.7 \mathrm{eV}$. One explanation for the large red shift observed from the original spectra taken before the anneal, along with the increased current yield as a function of anneal, is a charged, thinning-oxide model. Trapped charge at the surface explains the spectral offset, and the subsequent loss of charge as a function of anneal leads to the observed red-shift. The thinning oxide explains the net increase in current yield as a function of anneal. To explore this option we have modeled an oxide thinning from $0.9 \mathrm{~nm}$ initially down to $0.5 \mathrm{~nm}$.

For simplicity, the charge is assumed to be spread evenly throughout the oxide with a surface density around $5.5 \times 10^{12}$ electrons $/ \mathrm{cm}^{2}$, which is consistent with trap densities commonly found near $\mathrm{SiO}_{2}-\mathrm{Si}$ interfaces, especially for native oxides where the impurity concentration can be large at the surface. [18] The peak field strength of the trapped charge is taken to be $0.5 \mathrm{~V} / \mathrm{nm}$. As this field is an order of magnitude below that due to the laser, the effects of this field on emission probability is neglected. We have also assumed that the changing oxide thickness does not significantly alter the field enhancement factor, $\alpha$. The results of this model are shown in Figure 7.

Though simple, the model qualitatively reproduces all three major trends as observed experimentally. First, the initial decrease and then eventual increase in current yield as a function of thinning oxide. This is a result of the changing potential barrier profile as a function of oxide thickness which, at certain $d_{o x}$ values, can lead to higher emission when the oxide is increased while keeping the applied field fixed. Secondly, the presence and reduction of surface charge easily explains the $4-5 \mathrm{eV}$ shift of the energy spectra as observed in the experiment. However, it is clear that the effect of the oxide thinning occurs in the experimental data before a significant red-shift. This is consistent with the charge being located nearer to the interface of the $\mathrm{Si}_{-}-\mathrm{SiO}_{2}$. Finally, due to the extension of 


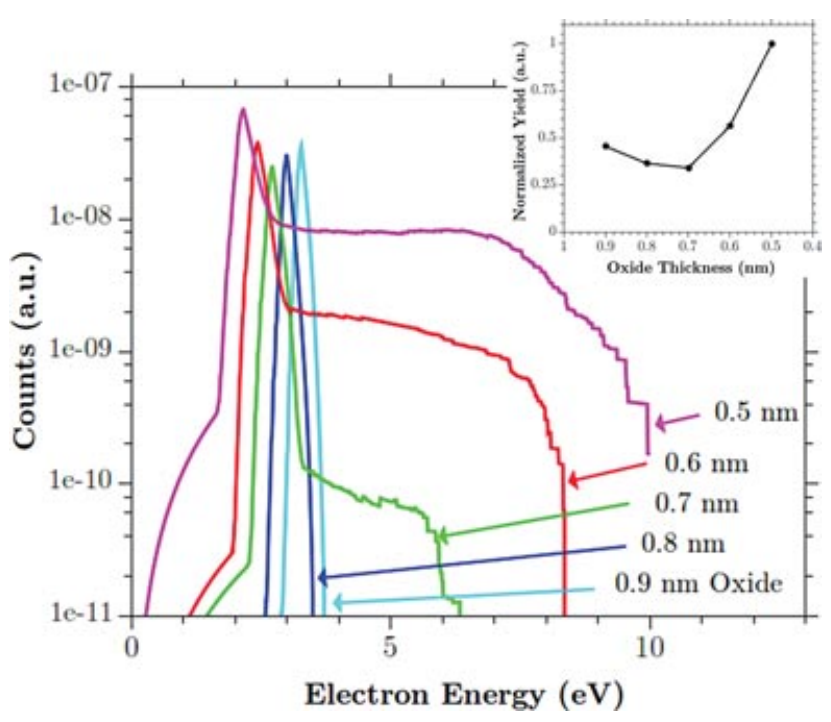

Figure 7 (online color at: www.ann-phys.org) Simulation results for a tip-model including a charged, thinning oxide. Three basic trends are reproduced in the model. First the prevalent red-shift is introduced due to the reduction of surface charge. Secondly, it accurately models the plateau suppression before anneal. Thirdly, the thinning-oxide models first a reduction and then increase in net electron yield. The inset shows the modeled total electron yield as a function of decreasing oxide thickness.

the tunnel exit away from the tip surface, the electron re-scattering is reduced thus suppressing the highenergy plateau before the anneal.

Despite explaining these features, it is clear that more work has to be done to understand all of the underlying dynamics at play during the annealing process more fully. One striking difference between the experiment and simulations is the significantly broader plateau observed for intermediate anneal times in the experimental data that is not reproduced in the simulation.

\section{Conclusions}

The energy distributions of photo-emitted electrons from a Si FEA composed of sharp conical tips has been presented. Without first removing the native oxide of the emitters, it was found that a laser treatment process could be used to activate the tips, increasing total electron yield. A high energy plateau in the energy distributions, as well as a slope change in the total current yield as a function of incident energy both indicate strongfield tunneling emission and electron re-scattering from the tips.

To further verify the analysis, a model was created using the "simple man" approach. The results of the model show good qualitative agreement with the experimental data. A sharp spectral peak is observed due to direct electron emission, and a broad energy plateau is present due to electron re-scattering from the tip. A rough fitting of the scattering probability gives a value of around $R=0.3$. The field enhancement due to the tips was found to be between 10-14, leading to peak fields ranging from 11$15.4 \mathrm{~V} / \mathrm{nm}$.

To replicate the effects of a red-shift and increased current yield as a function of anneal, a charged, thinningoxide model was used. The charge, most likely due to electron traps near the interface of this $\mathrm{Si}_{-} \mathrm{SiO}_{2}$, provides a push to the electrons accounting for the increased energy of the spectral peak before anneal, while the thinning oxide accounts for the change in tunneling rate with applied field. Reduction of oxide shows first a decrease in electron yield before eventual increase, which is confirmed by experimental observations. A surface charge density of around $5.5 \times 10^{12}$ electrons $/ \mathrm{cm}^{2}$ was found to sufficiently account for the net energy shift of the electron spectra before anneal.

Further work is still needed to verify the validity of the charged, thinning-oxide model, and the underlying processes at play. Future tests with a controlled level of oxide thickness are planned, along with carefully marked samples for post imaging of the surface to account for any structural changes that may take place. Given the success of the simple models presented here, FEA designs are being constructed that seek to reduce or avoid electron recollision altogether, thus reducing the broad plateau and leading to a more monoenergetic electron source.

Acknowledgements. This work was funded by Defense Advanced Research Projects Agency (DARPA)/Microsystems Technology Office and the Space and Naval Warfare Systems Center (SPAWAR) under contract N66001-11-1-4192 (DARPA Program Manager Tayo Akinwande and SPAWAR Program Manager Paul de la Houssaye). The content of this paper does not necessarily reflect the position or the policy of the US Government and no official endorsement of the US Government should be inferred. P.D. Keathley would like to acknowledge financial support from the Department of Defense through the National Defense Science and Engineering Fellowship, A. Sell acknowledges funding by a Feodor-Lynen fellowship of the Alexander von Humboldt Foundation, and W. Putnam acknowledges support by a NSF Graduate Research Fellowship.

Key words. Strong-Field Emission, Ultrafast Phenomena, Field Emitter Arrays.

\section{References}

[1] M. Kruger, M. Schenk, and P. Hommelhoff, Nature 475(7354), 78-81 (2011). 
[2] C. Ropers, D. R. Solli, C. P. Schulz, C. Lienau, and T. Elsaesser, Physical Review Letters 98(4), 043907 (2007).

[3] P. Hommelhoff, Y. Sortais, A. Aghajani-Talesh, and M. A. Kasevich, Physical Review Letters 96(7), 077401 (2006).

[4] P. Hommelhoff, C. Kealhofer, and M. A. Kasevich, Physical Review Letters 97(24), 247402 (2006).

[5] G. Herink, D. R. Solli, M. Gulde, and C. Ropers, Nature 483(7388), 190-193 (2012).

[6] A. Anghel, F. Ardana-Lamas, F. Le Pimpec, and C. P. Hauri, Physical Review Letters 108(19), 194801 (2012).

[7] A. Mustonen, P. Beaud, E. Kirk, T. Feurer, and S. Tsujino, Applied Physics Letters 99(10), 103504-103504-3 (2011).

[8] P. Baum and A. H. Zewail, Proceedings of the National Academy of Sciences 103(44), 16105-16110 (2006).

[9] W. S. Graves, F. X. Kärtner, D. E. Moncton, and P. Piot, Physical Review Letters 108(26), 263904 (2012).

[10] R. Bormann, M. Gulde, A. Weismann, S. V. Yalunin, and C. Ropers, Physical Review Letters 105(14), 147601 (2010).

[11] J. T. Krug, E. J. Sánchez, and X. S. Xie, The Journal of Chemical Physics 116(24), 10895-10901 (2002).
[12] L. Velásquez-García, S. Guerrera, Y. Niu, and A. Akinwande Electron Devices, IEEE Transactions on 58(6), 1775-1782 (2011).

[13] L. Velásquez-García, S. Guerrera, Y. Niu, and A. Akinwande Electron Devices, IEEE Transactions on 58(6), 1783-1791 (2011).

[14] M. Schenk, M. Krüger, and P. Hommelhoff, Physical Review Letters 105(25), 257601 (2010).

[15] S. V. Yalunin, M. Gulde, and C. Ropers, Physical Review B 84(19), 195426 (2011).

[16] Y. C. Martin, H. F. Hamann, and H. K. Wickramasinghe, Journal of Applied Physics 89, 5774 (2001).

[17] W. Becker, F. Grasbon, R. Kopold, D. Milošević, G. Paulus, H. Walther, B. Bederson, and H. Walther, Above-threshold ionization: From classical features to quantum effects, in: Advances In Atomic, Molecular, and Optical Physics, (Academic Press, 2002), pp. 3598.

[18] Q. Huang, Journal of Applied Physics 79(7), 3703-3707 (1996).

[19] Q. Huang, Journal of Applied Physics 78(11), 6770 (1995).

[20] M. Krüger, M. Schenk, M. Förster, and P. Hommelhoff, Journal of Physics B: Atomic, Molecular and Optical Physics 45(7), 074006 (2012). 